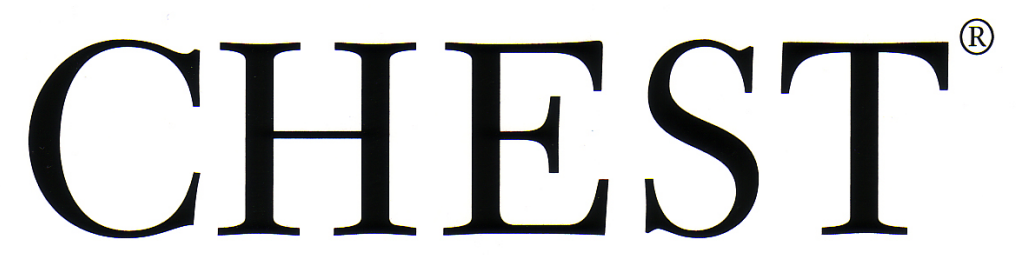

\author{
THE CARDIOPULMONARY \\ AND CRITICAL CARE JOURNAL \\ FOR PULMONOLOGISTS, CARDIOLOGISTS, CARDIOTHORACIC SURGEONS, \\ CRITICAL CARE PHYSICIANS, AND RELATED SPECIALISTS
}

\title{
Acute Effects of Exercise in Patients With Previous Deep Venous Thrombosis: Impact of the Postthrombotic Syndrome
}

Susan R. Kahn, Laurent Azoulay, Andrew Hirsch, Michael Haber, Carla Strulovitch and Ian Shrier

Chest 2003;123;399-405

DOI: $10.1378 /$ chest.123.2.399

This information is current as of May 16, 2006

The online version of this article, along with updated information and services, is located on the World Wide Web at:

http://www.chestjournal.org/cgi/content/full/123/2/399

CHEST is the official journal of the American College of Chest Physicians. It has been published monthly since 1935. Copyright 2005 by the American College of Chest Physicians, 3300 Dundee Road, Northbrook IL 60062. All rights reserved. No part of this article or PDF may be reproduced or distributed without the prior written permission of the copyright holder. ISSN: 0012-3692.

A M E R C A N C O L L E E O F

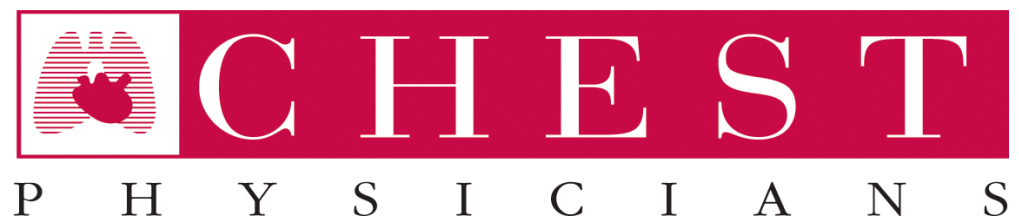




\title{
Acute Effects of Exercise in Patients With Previous Deep Venous Thrombosis*
}

\section{Impact of the Postthrombotic Syndrome}

\author{
Susan R. Kahn, MD, MSc; Laurent Azoulay, BSc; Andrew Hirsch, MD;
}

Michael Haber, BSc; Carla Strulovitch, RN; and Ian Shrier, MD, PhD

\begin{abstract}
Background: The postthrombotic syndrome (PTS) occurs frequently after deep venous thrombosis (DVT) and is believed to worsen with upright posture and physical activity. However, the effects of exercise in patients with previous DVT have not been studied.

Study objectives: To determine whether previous DVT and PTS limit the ability to exercise, and whether exercise increases the severity of venous symptoms and signs.

Design and setting: A repeated-measures cohort study that was conducted at a universityaffiliated teaching hospital, 1999-2000.

Participants: Subjects with a first episode of unilateral DVT at least 1 year earlier were recruited from the Thrombosis Clinic (total, 41 subjects; with PTS, 19 subjects).

Intervention: Treadmill exercise session.

Measurements and results: Venous symptoms, calf muscle fatigability, flexibility, and leg volume before and after treadmill exercise were measured and compared. Exercise did not worsen venous symptoms, despite a higher gain in affected leg volume in subjects with PTS vs subjects without PTS (mean difference: affected leg, $53 \mathrm{~mL}$; unaffected leg, $-15 \mathrm{~mL} ; \mathrm{p}=0.018$ ). Calf flexibility significantly improved after exercise in subjects with PTS (gastrocnemius: affectedunaffected, PTS vs no PTS $+4.5^{\circ}, \mathrm{p}=0.0029$; soleus: affected-unaffected, PTS vs no PTS $+5.7^{\circ}$, $\mathbf{p}=\mathbf{0 . 0 0 1 1 )}$.

Conclusions: Exercise did not acutely exacerbate symptoms and, in subjects with PTS, resulted in improved flexibility in the affected leg. Our findings suggest that treadmill or similar exercise is unlikely to make symptoms of PTS worse, and may improve flexibility. Further study is indicated to determine whether a regular exercise-training program might have a role in the management of patients with PTS, since, to date, the treatment options for this condition are limited.
\end{abstract}

(CHEST 2003; 123:399-405)

Key words: deep venous thrombosis; edema; exercise; posthrombotic syndrome; symptoms

Abbreviations: DVT $=$ deep venous thrombosis; PTS $=$ postthrombotic syndrome

D eep venous thrombosis (DVT) is a common disorder with an incidence rate of approximately 1 case per 1,000 persons per year. ${ }^{1}$ Postthrombotic syndrome (PTS), which is a chronic condition consisting of leg pain, edema, venous ectasia, skin induration, and ulceration, occurs in approximately 20 to $50 \%$ of patients with DVT and

${ }^{*}$ From the Center for Clinical Epidemiology and Community Studies (Drs. Kahn and Shrier, Mr. Azoulay, Ms. Strulovitch, and Mr. Haber) and the Department of Medicine (Dr. Hirsch), Sir Mortimer B. Davis Jewish General Hospital, McGill University, Montréal, Canada.

Drs. Kahn and Shrier are Chercheurs Boursier Clinicien (Clinical Research Scholars) supported by the Fonds de Recherche en Santé du Québec. This study was supported by an unrestricted grant-in-aid from the Beiersdorf-Jobst Research Program of the American College of Phlebology. usually becomes established within the first 2 years after the acute thrombotic episode..$^{2,3}$ Since DVT affects adults of all age groups, and the incidence of

For editorial comment see page 327

associated PTS is high, the population burden of PTS is considerable. Despite its high prevalence and associated morbidity, the impact of PTS on the daily

Manuscript received April 24, 2002; revision accepted August 16, 2002.

Correspondence to: Susan R. Kahn, MD, MSc, Center for Clinical Epidemiology and Community Studies, Sir Mortimer B. Davis Jewish General Hospital, 3755 Cote Ste Catherine, Rm A-127, Montreal, QU H3T 1E2, Canada; e-mail: susan.kahn@mcgill.ca 
activities of affected patients has received little attention. ${ }^{4}$ For example, many DVT patients are otherwise healthy and eager to resume exercise programs, but symptoms of PTS are believed to worsen with walking and upright posture. ${ }^{5}$ To date, however, the effects of exercise in patients with previous DVT have not been studied. Furthermore, the impact of PTS on the ability to exercise and, conversely, the impact of exercise on PTS symptoms and signs are not known.

The objectives of this study were to examine, among patients with previous DVT, (1) whether the presence of PTS limits the ability to perform exercise and (2) whether exercise increases the severity of venous symptoms and signs acutely.

\section{Materials AND Methods}

\section{Subjects}

Study subjects were recruited from the Thrombosis Clinic of our hospital, a clinic that observes all patients in whom venous thromboembolism had been diagnosed. Sequential patients with a first diagnosis of unilateral DVT established by objective testing at least 1 year prior were approached for participation in the study. Subjects were excluded if they had bilateral DVT, had experienced more than one episode of DVT, had symptomatic pulmonary embolism, had any medical condition that precluded treadmill exercise, were incapable of self-administering a questionnaire in French or English, or were unable to or had refused to provide informed consent. Prior to its initiation, the Research Ethics Committee of the hospital approved the study.

\section{Study Procedures}

The study protocol had three components, all of which took place during a single study visit, as follows: baseline (preexercise) measures; an exercise protocol; and postexercise measures.

\section{Baseline Measures}

Data were collected on age, gender, objective test used to diagnose DVT, and site of DVT from the subjects' hospital records by one of two research assistants. Proximal DVT was defined as DVT involving the popliteal or more proximal venous segments, with or without associated calf (distal) DVT. The term affected leg indicates the leg in which the DVT occurred.

One of three study physicians, all of whom were blinded to the data from the hospital record, supervised each experiment and ascertained the diagnosis and severity of PTS. We used the PTS scale of Villalta et al, ${ }^{6}$ which assigns a severity score from 0 (not present or minimal) to 3 (severe) for five symptoms ( $i e$, pain, cramps, heaviness, pruritus, and paresthesia) and six signs (ie, edema, skin induration, hyperpigmentation, venous ectasia, redness, and pain during calf compression). A summative score of $\leq 4$ indicates the absence of PTS, a score of 5 to 14 indicates mild/moderate PTS (note: the scale does not separate mild and moderate categories), and a score of $\geq 15$ or the presence of a venous ulcer indicates severe PTS. This scale has high interobserver agreement. ${ }^{6}$

To assess usual, habitual physical activity, subjects completed the Godin Leisure Time Questionnaire. ${ }^{7}$ Subjects then rated their usual severity of individual symptoms that are typically associated with venous disease (ie, heaviness, aching, swelling, heat or burning, restless legs, throbbing, itching, and tingling $)^{8}$ using 10 -cm visual analog scales.

Leg muscle fatigability, flexibility, and volume then were measured for both legs in order that the unaffected leg could serve as a control for the affected leg. These measures were chosen because fatigability and reduced flexibility are associated with exercise-induced symptoms such as stiffness and cramping in healthy individuals, ${ }^{9,10}$ and leg swelling is a known consequence of DVT.

Fatigability of the calf muscles was assessed 30 min prior to the exercise test using a heel-lift test during which time the subject raised the heel $5 \mathrm{~cm}$ on a standardized device, held that position for $1 \mathrm{~s}$, and then lowered the heel. This was repeated at a cadence of 23 lifts per minute as long as the patient was able, and the total number of heel lifts was counted. This test was shown to have high intrarater reliability both in normal volunteers and in our study sample (Haber M, Azoulay L, Kahn SR, et al; unpublished observations; 2002) and is similar to fatigability tests that have been validated for the back and abdominal muscles. ${ }^{11,12}$

Calf muscle flexibility of the soleus muscle (ie, a postural muscle that is used in standing and walking) and the gastrocnemius muscle (ie, a nonpostural muscle that is used in running and jumping) was assessed with a gravity-based goniometer ${ }^{13,14}$ as follows. For the gastrocnemius muscle, the subject stood with the knee straight and the foot planted on the ground with the heel fixed and the toes pointing straight ahead. The patient moved the leg forward, keeping the heel firmly planted on the ground. Flexibility was defined as the angular rotation of the tibia away from the perpendicular $\left(0^{\circ}\right)$ to the floor during the maneuver. For the soleus muscle, the same procedure was used, but the knee was bent. For both maneuvers, a larger angle indicates greater flexibility.

Leg volume was measured by placing the lower leg into a standardized container of water and measuring the volume of water that was displaced. In order to assess the reliability of this instrument, in a separate experiment we repeated volume measurements 10 times in one subject. The SD of the measures $(74 \mathrm{~mL})$ represented $2.6 \%$ of the mean volume $(2,800 \mathrm{~mL})$, indicating good reliability.

\section{Exercise Protocol}

Patients exercised on a treadmill that was set at a 5\% incline. After a 5-min warm-up, subjects walked or ran at a speed that was comfortable and caused mild tachypnea and sweating. Subjects exercised for as long as they were able up to a maximum of 30 min. Thirty minutes was chosen because this duration of brisk walking each day will provide much of the total health benefits of exercise. ${ }^{15}$ The total treadmill time, the maximum speed, and the mean heart rate achieved were documented.

\section{Postexercise Measures}

After exercise, calf muscle flexibility and leg volume were remeasured in each leg. Subjects were asked again to score the severity of individual symptoms in each leg using the same visual analog scales, without access to their preexercise symptom scores.

\section{Sample Size Requirements}

The primary comparisons were between the affected and unaffected legs of the same subjects. In the absence of data from previous studies, we considered that a between-leg or after- 
before exercise difference in the means of one SD would be clinically relevant. Since all of our study outcomes were continuous variables, we calculated that in order to achieve a probability of type I error of 0.05 and a probability of type II error of 0.20 (power, 0.80), we required at least 17 subjects with PTS and 17 subjects without PTS.

\section{Statistical Analysis}

For the comparison of baseline measures in subjects with PTS vs those without PTS, a Fisher exact test of proportions, $t$ tests of means, or Mann-Whitney tests of medians were used, as appropriate. For within-patient comparisons of measures in the affected leg vs the unaffected leg at baseline or after exercise vs before exercise, paired $t$ tests of mean differences (affected unaffected and/or after - before) were used. For the measurement of association between continuous variables, Pearson correlation coefficients were calculated.

\section{RESULTS}

\section{Study Population}

Forty-three patients were recruited over a 1-year period (1999 to 2000). Two patients were excluded (one had a history of bilateral DVT and one was not able to follow the research assistant's instructions), leaving a study population of 41 subjects. PTS, as defined as a Villalta score of $\geq 5$, was present in 19 patients $(46.3 \%)$ in the study group, and the PTS was categorized as mild/moderate in 17 subjects and as severe in 2 subjects. The mean (SD) PTS score was 2.1 (1.3), 6.4 (1.3), and $19.0(0.0)$, respectively, in subjects with no PTS, mild/moderate PTS, and severe PTS. There were no statistically significant differences in age, sex distribution, site of index DVT, or number of years since DVT diagnosis among subjects with PTS vs those without PTS (Table 1).

\section{Baseline Measures}

Affected vs Unaffected Leg: Generally, symptoms were rated as mild (all were rated $<3 \mathrm{~cm}$ on a $10-\mathrm{cm}$

Table 1-Characteristics of Study Population

\begin{tabular}{llll}
\hline \hline \multicolumn{1}{c}{ Characteristics } & \multicolumn{1}{c}{ PTS } & No PTS & \\
\hline Age, ${ }^{*}$ yr & $51.7(12.3)$ & $50.2(15.0)$ & 0.72 \\
Female sex & $52.6 \%$ & $31.8 \%$ & 0.18 \\
Proximal DVT & $78.9 \%$ & $63.6 \%$ & 0.28 \\
Years since DVT & $2.4(1.4)$ & $2.0(1.4)$ & 0.39 \\
$\quad$ diagnosis* & & & \\
PTS severity, ${ }^{\dagger}$ No. of & & & \\
$\quad$ patients & & & \\
Mild/moderate & 17 & & \\
Severe & 2 & & \\
\hline
\end{tabular}

*Values given as mean (SD).

†Graded using Villalta scale. ${ }^{6}$ visual analog scale). However, subjects complained of significantly more heaviness, aching, swelling, and throbbing in their affected leg compared to their unaffected leg (Fig 1).

Baseline measures of fatigability, flexibility, and leg volume of the entire study population are presented in Table 2. Subjects demonstrated greater fatigability in their affected leg vs their unaffected leg, as reflected by an ability to perform $12 \%$ fewer heel lifts $(p=0.02)$. Leg volume was an average of $60 \mathrm{~mL}$ higher in the affected leg vs the unaffected leg $(p=0.046)$. No differences in calf flexibility between affected and unaffected legs were noted. There were no correlations between fatigability of the affected leg and (1) flexibility or (2) volume of the affected leg (data not shown).

PTS vs No PTS: Compared to subjects without PTS, not unexpectedly, those with PTS had higher mean scores in their affected leg for all symptoms, which were statistically significant for swelling and tingling (Fig 2). Differences in baseline measures in subjects with and without PTS are given in Table 3 . There were no differences in the weekly habitual activity scores between groups. The mean $( \pm \mathrm{SD})$ score of $26.8 \pm 18.0$ for all subjects corresponds to the following: three sessions per week of $\geq 15 \mathrm{~min}$ of strenuous activity; five sessions of moderate activity; or nine sessions of light activity. The magnitude of the difference in fatigability between the affected and unaffected legs was similar in subjects with and without PTS $(p=0.56)$. However, among subjects with PTS, considering the affected leg only, those with mild/moderate PTS $(\mathrm{n}=17)$ performed a mean of $30.6 \pm 21.7$ heel lifts compared to $20.5 \pm 3.5$ heel lifts in subjects with severe PTS $(\mathrm{n}=2)$ [no $\mathrm{p}$ values were obtained due to the small numbers of subjects in the severe PTS group].

Subjects with PTS had reduced calf muscle flexibility in their affected legs compared to subjects without PTS (Table 3). For the gastrocnemius muscle, subjects with PTS had a mean $3.1 \pm 4.4^{\circ}$ less flexibility in their affected vs unaffected legs, while subjects without PTS had $0.7 \pm 5.4^{\circ}$ more flexibility in their affected leg vs their unaffected leg $(\mathrm{p}=0.02$ [difference between PTS and no PTS]). For the soleus muscle, subjects with PTS had $2.2 \pm 4.0^{\circ}$ less flexibility in their affected leg vs their unaffected leg, while subjects without PTS had $0.8 \pm 5.9^{\circ}$ more flexibility in their affected leg vs their unaffected leg $(\mathrm{p}=0.07$ [difference between PTS and no PTS]). There was a trend to higher leg volume in the affected leg compared to the unaffected leg in subjects with PTS vs subjects without PTS (mean difference: affected leg, $120 \pm 240 \mathrm{~mL}$; unaffected leg, $10 \pm 120 \mathrm{~mL} ; \mathrm{p}=0.068)$. There were no signif- 


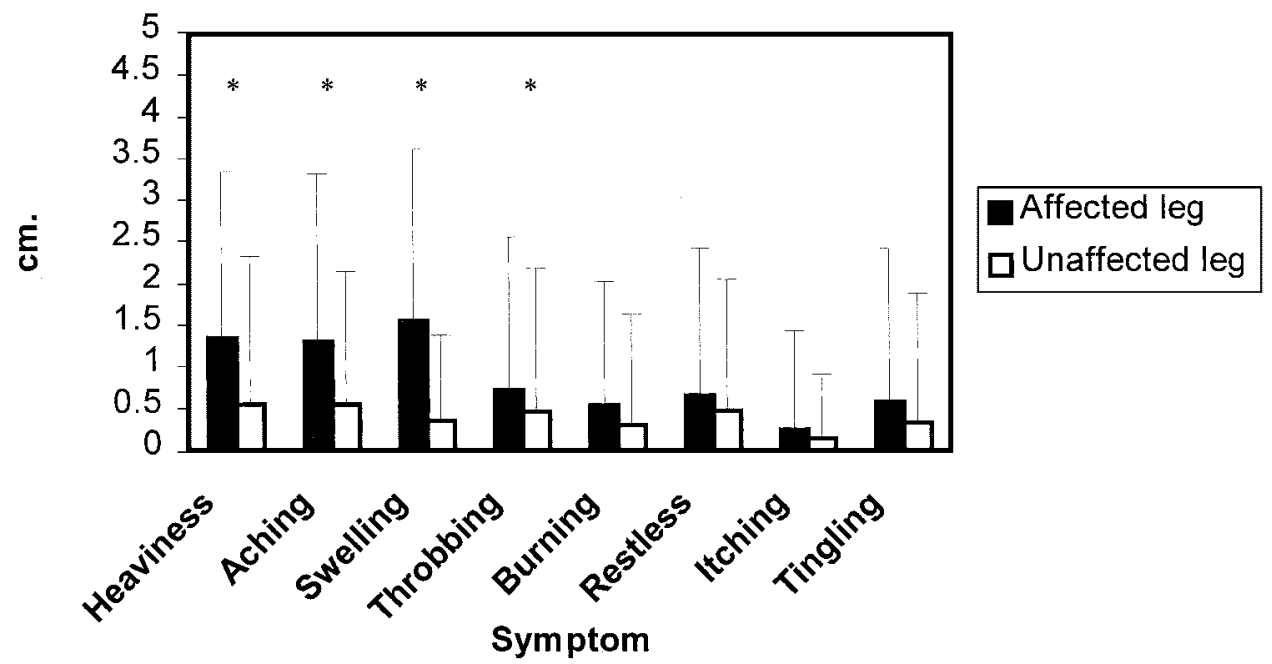

FIGURE 1. Symptom severity at baseline (mean $\pm \mathrm{SD}$ ) in the study population (affected leg vs unaffected leg). $*=$ heaviness, $p=0.001$; aching, $p=0.0024$; swelling, $p=0.0002$; throbbing, $\mathrm{p}=0.04$ (all other comparisons were nonsignificant).

icant correlations between the severity of PTS (as assessed by the summative PTS score) and betweenleg differences in flexibility or leg volume (data not shown).

\section{Exercise Protocol}

The mean treadmill speed at which subjects exercised was $3.2 \pm 0.9$ miles per hour. The mean heart rate achieved by subjects was $131.2 \pm 19.6$ beats/ min, or $78 \%$ of the age-predicted maximum heart rate (calculated as 220 - age). There were no differences in these measures in subjects with PTS vs those without PTS; however, there was a trend toward lower median treadmill times in subjects with PTS (median, $18.4 \mathrm{~min}$; interquartile range, 21.5 min) vs subjects without PTS (median, $30.0 \mathrm{~min}$; interquartile range, $12.2 \mathrm{~min} ; \mathrm{p}=0.15$ ). There were no correlations between the fatigability of the affected leg at baseline and (1) treadmill speed or (2) treadmill duration (data not shown).

Table 2-Baseline (Preexercise) Measures in Affected vs Unaffected Legs for the Entire Study Population*

\begin{tabular}{lcc}
\hline \hline \multicolumn{1}{c}{ Variables } & Affected Leg & Unaffected Leg \\
\hline Fatigability, No. of heel lifts $\uparrow$ & $30.3 \pm 17.2$ & $33.9 \pm 19.5$ \\
Leg volume, $\mathrm{t} \mathrm{mL}$ & $2,900 \pm 710$ & $2,840 \pm 660$ \\
Flexibility & & \\
$\quad$ Gastrocnemius muscle & $24.5 \pm 8.0^{\circ}$ & $25.7 \pm 7.6^{\circ}$ \\
$\quad$ Soleus muscle & $31.2 \pm 6.8^{\circ}$ & $31.8 \pm 7.8^{\circ}$ \\
\hline
\end{tabular}

*Values given as mean $\pm \mathrm{SD}$.

$\uparrow \mathrm{p}=0.02$ (affected vs unaffected legs).

$+\mathrm{p}=0.046$ (affected vs unaffected legs).

\section{Postexercise Measures}

Symptoms: Overall, there were no changes in individual leg symptoms in either the affected leg or the unaffected leg following exercise. After-before exercise differences in symptom ratings (in centimeters) ranged from -0.01 to +0.52 (difference not significant) in the affected leg, and from -0.04 to +0.44 (difference not significant) in the unaffected leg, and were not influenced by the presence or severity of PTS. There were no correlations between baseline fatigability in the affected leg and the change in individual symptoms in the affected leg vs the unaffected leg with exercise (data not shown).

Leg Volume and Flexibility: Leg volume increased with exercise both in unaffected legs (mean increase from preexercise, $59 \pm 82 \mathrm{~mL} ; \mathrm{p}<0.0001)$ and affected legs (mean increase from preexercise, $76 \pm 110 \mathrm{~mL} ; \mathrm{p}<0.0001)$. However, subjects with PTS gained significantly more volume in their affected legs compared to their unaffected legs compared to subjects without PTS (mean between-leg difference, $53 \pm 108$ vs $-15 \pm 64 \mathrm{~mL}$, respectively; $\mathrm{p}=0.018)$. Furthermore, subjects with mild/moderate PTS gained a mean of $37 \pm 79 \mathrm{~mL}$ in volume, whereas those with severe PTS gained a mean of $187 \pm 265 \mathrm{~mL}$ in volume (no $\mathrm{p}$ value was obtained due to the small numbers of subjects in the severe PTS group).

In subjects with PTS, gastrocnemius muscle flexibility improved significantly after exercise in the affected leg vs that in the unaffected leg compared to subjects without PTS (mean difference, 4.5; 


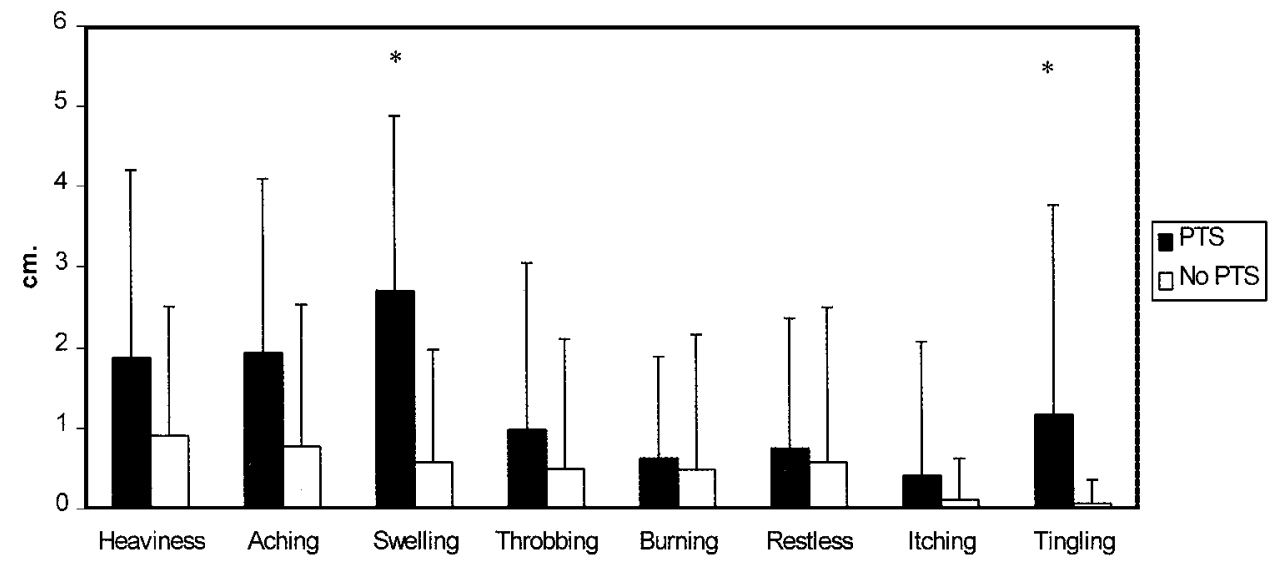

FigURE 2. Symptom severity at baseline (mean $\pm \mathrm{SD})$ in the affected leg in subjects with PTS $(\mathrm{n}=19)$ and without PTS $(\mathrm{n}=22)$. $*=$ swelling, $\mathrm{p}=0.0006$; tingling, $\mathrm{p}=0.05$ (all other comparisons were nonsignificant).

$\mathrm{p}=0.0029$ ), as did soleus muscle flexibility (mean difference, $5.7^{\circ} ; \mathrm{p}=0.0011$ ).

We explored whether the postexercise improvement in flexibility in subjects with PTS compared to subjects without PTS could be explained, physiologically, by less gain in leg volume among those subjects whose flexibility improved. However, there was no demonstrable correlation between change in leg volume and change in gastrocnemius muscle flexibility $\left(R^{2}=0.03\right)$ or soleus muscle flexibility $\left(R^{2}=0.07\right)$. Correlation between these variables was also absent when stratifying the population by PTS vs no PTS (data not shown).

Table 3-Baseline (Preexercise) Measures in All Subjects*

\begin{tabular}{lcc}
\hline \hline \multicolumn{1}{c}{ Variables } & $\begin{array}{c}\text { PTS } \\
(\mathrm{n}=19)\end{array}$ & $\begin{array}{c}\text { No PTS } \\
(\mathrm{n}=21)\end{array}$ \\
\hline $\begin{array}{l}\text { Habitual activity score } \\
\text { Fatigability (heel lifts) }\end{array}$ & $26.9 \pm 18.1$ & $26.8 \pm 18.3$ \\
$\quad$ Affected leg & $29.6 \pm 20.8$ & $30.9 \pm 13.6$ \\
$\quad$ Unaffected leg & $32.4 \pm 20.8$ & $35.4 \pm 18.6$ \\
Flexibility & & \\
$\quad$ Gastrocnemius muscle + & & \\
$\quad$ Affected leg & $20.6 \pm 8.0^{\circ}$ & $28.0 \pm 6.4^{\circ}$ \\
$\quad$ Unaffected leg & $23.7 \pm 8.1^{\circ}$ & $27.3 \pm 6.9^{\circ}$ \\
$\quad$ Soleus muscle§ & & \\
$\quad$ Affected leg & $27.9 \pm 6.0^{\circ}$ & $34.0 \pm 6.3^{\circ}$ \\
$\quad$ Unaffected leg & $30.1 \pm 7.7^{\circ}$ & $33.2 \pm 7.7^{\circ}$ \\
Leg volume, $\| \mathrm{L}$ & & \\
$\quad$ Affected leg & & \\
$\quad$ Unaffected leg & $3.02 \pm .89$ & $2.81 \pm .51$ \\
\hline
\end{tabular}

*Values given as mean $\pm \mathrm{SD}$.

† As assessed by the Godin Leisure Time Questionnaire. ${ }^{7}$

$+\mathrm{p}=0.02$ (affected-unaffected legs, PTS vs no-PTS patients.

$\S \mathrm{p}=0.07$ (affected-unaffected legs, PTS vs no-PTS patients.

$\| p=0.068$ (affected-unaffected legs, PTS vs no-PTS patients.

\section{Discussion}

DVT and its chronic sequela, PTS, affect adults of all age groups, many of whom are active and eager to exercise. The effects of exercise on patients with DVT and PTS have not been studied previously. From a physiologic point of view, exercise could either help or worsen PTS. During exercise, muscle contraction increases the pressure outside of the veins and propels blood back to the heart (calf muscle pump), reducing the hydrostatic pressure gradient required for edema formation. ${ }^{16}$ However, due to a decrease in arteriolar resistance, muscle blood flow increases above control conditions between contractions (active hyperemia).17,18 Normally, the venous system easily accommodates this increased flow, but venous obstruction or reflux, which can occur after DVT, ${ }^{5}$ might raise venous pressure to the extent that outflow cannot match inflow. The resultant increased volume within the vascular bed could raise capillary pressure, promote fluid transudation from the capillaries into the interstitial space, and impair leg muscle perfusion. ${ }^{19,20}$ These phenomena could promote exercise-induced leg swelling, and, potentially, the worsening of venous symptoms due to both edema and muscle fatigue.

Our study consisted of baseline measures, an exercise protocol, and postexercise measures in subjects with previous DVT, approximately half of whom had PTS. Regardless of whether or not subjects had PTS, their affected legs were significantly more symptomatic at baseline (ie, heaviness, aching, swelling, and throbbing) than their unaffected legs, indicating that even among DVT patients who did not meet the criteria for PTS, persistent symptoms in the affected leg were common. In addition, the 
affected leg had higher volume and was more fatigable than the unaffected leg, and the two subjects with severe PTS were more fatigable in their affected leg than those with mild/moderate PTS. Fatigability in the affected leg may be due to the combined effects of reduced muscle perfusion, resulting from venous hypertension, ${ }^{19,20}$ and of leg swelling, which directly increases leg weight, further increasing the demands placed on the calf muscles with resultant muscle deconditioning and fatigability. Although leg muscle function following DVT has not, to our knowledge, previously been studied, there is evidence from histologic studies ${ }^{21,22}$ that muscle is damaged by acute or chronic venous hypertension. This could lead to muscle weakness and decreased endurance.

We found several differences in the baseline (ie, preexercise) measures in our subjects with PTS compared to those without PTS. When comparing affected legs to unaffected legs, subjects with PTS had decreased soleus and gastrocnemius muscle flexibility and a trend to higher leg volume compared to subjects without PTS. However, subjects with and without PTS had similar, moderate, levels of frequency and intensity of weekly habitual exercise, suggesting that these differences in flexibility and leg volume did not significantly limit the ability to perform exercise of mild or moderate intensity. That baseline habitual activity was similarly mild to moderate in intensity in subjects with PTS vs those without PTS has not, to our knowledge, been described previously. This could indicate that, despite PTS, patients can carry out low levels of habitual activity, or it may reflect that PTS subjects with even lower levels of habitual activity chose not to enroll in the study. However, the comparable baseline activity in subjects with and without PTS suggests that differences in the habitual activity level are unlikely to explain our findings.

We found that subjects with PTS were able to achieve the same treadmill speeds and mean heart rates as those without PTS, but there was a trend to shorter treadmill time, suggesting that patients with PTS may not be able to exercise for as long a duration as those without PTS. The reasons for this are uncertain but could be due, in part, to the higher leg volumes and/or reduced flexibility in subjects with PTS.

Exercise resulted in significant gains in leg volume in both the affected and unaffected legs of subjects, but the between-leg gain (ie, affected - unaffected gain) was significantly higher among subjects with PTS vs those without PTS. These gains in volume did not, however, result in a worsening of symptoms with exercise. We also found that exercise acutely improved the flexibility of both the gastrocnemius and soleus muscles to a significant degree in affected limbs compared to that in the unaffected limbs of subjects with PTS, but not in subjects without PTS. The lack of increased flexibility after exercise in the unaffected legs of our study subjects is consistent with the results of previous studies of control subjects. ${ }^{23}$

Subjects in our study underwent a single exercise session, hence we are unable to comment on the expected effects of regular, longer term exercise training on leg volume and calf flexibility in PTS patients. Our findings, while preliminary, suggest the possibility that treadmill or similar exercise might have beneficial effects in PTS patients. Whether a standardized exercise-training program to strengthen and increase the flexibility of calf muscles could lead to more efficient calf pump function is not known, but it merits further study since, to date, interventions aimed at improving the condition of patients with established PTS have proven disappointing. ${ }^{4}$ The beneficial effects of exercise therapy for chronic arterial lower limb claudication are supported by a number of studies. ${ }^{24,25}$ The putative mechanisms for these effects include blood flow redistribution, improved blood rheology, enhanced biomechanical efficiency, and changes in pain perception. ${ }^{26}$ Whether these mechanisms also might be operative in the case of patients with chronic venous hypertension of the lower limb is not known.

Our study has several limitations. There is no objective, "gold standard" test with which to diagnose PTS ${ }^{4}$; however, we used a validated clinical scale to diagnose and establish the severity of PTS. This scale has been used in prospective studies of PTS. ${ }^{2,3}$ Nevertheless, it is possible that misclassification of the diagnosis or severity of PTS may have occurred for some subjects. Our study population consisted of patients attending our Thrombosis Clinic who agreed to participate and were able to participate in this study, hence we may have selected for less severely affected patients, since those with more severe symptoms at baseline or those with symptoms that worsen with exercise might have declined to participate in the study. This could limit the generalizability of our findings to PTS patients with a broader spectrum of severity. Also, because we were interested in exercise duration at recommended intensities of exercise, we did not use a fixed duration, speed, or standardized increasing intensity in our treadmill exercise protocol. We were interested in whether exercise of recommended intensity would increase symptoms, not whether maximal exercise induced symptoms. By allowing a selfselected pace, we were able to determine whether the amount of exercise the subject would normally achieve led to a worsening of symptoms and signs in that subject. Our use of the unaffected leg as a 
control allowed us to control for different treadmill speed and duration among subjects. Furthermore, all patients sweated during the test, and the mean heart rate was $78 \%$ of the age-predicted maximum heart rate, suggesting that the patients exercised at a moderate intensity. Finally, our study was not designed to address whether exercise has effects on DVT prevention or formation.

In conclusion, in a group of middle-aged, moderately active patients with prior DVT, venous symptoms were worse in the affected leg compared to the unaffected leg, and subjects with PTS had, at baseline, higher leg volume, lower flexibility, and more severe venous symptoms compared to subjects without PTS. Despite this, the treadmill exercise protocol that was used in this study did not worsen symptoms. Furthermore, in subjects with PTS, exercise resulted in significantly improved gastrocnemius and soleus muscle flexibility in the affected compared to the unaffected leg compared to subjects without PTS. Our results suggest that physicians can endorse exercise for their DVT and PTS patients as an activity that is unlikely to worsen venous symptoms and that may lead to benefits such as improved flexibility. Furthermore, our results provide an impetus to study the potential role of a longer term exercise program that combines elements of strength and flexibility training in the management of PTS, a condition for which, to date, treatment options have been limited.

\section{REFERENCES}

1 Anderson FA, Wheeler B, Goldberg RJ, et al. A populationbased perspective of the hospital incidence and case-fatality rates of deep vein thrombosis and pulmonary embolism. Ann Intern Med 1991; 151:933-938

2 Brandjes DPM, Buller HR, Heijboer H, et al. Randomized trial of effect of compression stockings in patients with symptomatic proximal-vein thrombosis. Lancet 1997; 349: 759-762

3 Prandoni $\mathrm{P}$, Lensing AWA, Cogo A, et al. The long-term clinical course of acute deep venous thrombosis. Ann Intern Med 1996; 125:1-7

4 Kahn SR, Solymoss S, Lamping DL, et al. Long-term outcomes after deep vein thrombosis: post-phlebitic syndrome and quality of life. J Gen Intern Med 2000; 15:425-429

5 Kearon C, Salzman EW, Hirsh J. Epidemiology, pathogenesis and natural history of venous thrombosis. In: Colman RW, Hirsh J, Marder VJ, et al, eds. Hemostasis and thrombosis: basic principles and clinical practice. 4th ed. Philadelphia, PA: Lippincott-Williams \& Wilkins, 2001; 1153-1177

6 Villalta S, Bagatella P, Piccioli A, et al. Assessment of validity, and reproducibility of a clinical. scale for the post-thrombotic syndrome [abstract]. Haemostasis 1994; 24(suppl):158a

7 Godin G, Shephard RJ. A Simple method to assess exercise behavior in the community. Can J Appl Sport Sci 1985; 10:141-146

8 Kurz X, Kahn SR, Abenhaim L, et al. Chronic venous disorders of the leg: epidemiology, outcomes, diagnosis and management: summary of an evidence-based report of the VEINES task force. Int Angiol 1999; 18:83-102

9 Mair SD, Seaber AV, Glisson RR, et al. The role of fatigue in susceptibility to acute muscle strain injury. Am J Sports Med 1996; 24:137-143

10 Schwellnus MP, Derman EW, Noakes TD. Aetiology of skeletal muscle 'cramps' during exercise: a novel hypothesis. J Sports Sci 1997; 15:277-285

11 Ito T, Shirado O, Suzuki H, et al. Lumbar trunk muscle endurance testing: an inexpensive alternative to a machine for evaluation. Arch Phys Med Rehabil 1996; 77:75-79

12 Risch SV, Norvell NK, Pollock ML, et al. Lumbar strengthening in chronic low back pain patients: physiologic and psychological benefits. Spine 1993; 18:232-238

13 Boone DC, Azen SP, Lin CM, et al. Reliability of goniometric measurements. Phys Ther 1978; 58:1355-1390

14 Gajdosik RL, Bohannon RW. Clinical measurement of range of motion: review of goniometry emphasizing reliability and validity. Phys Ther 1987; 67:1867-1872

15 NIH consensus statement. In: Leon AS, ed. Physical activity and cardiovascular health: a national consensus. Champaign, IL: Human Kinetics, 1997; 3-13

16 Stick C, Grau H, Witzleb E. On the edema-preventing effect of the calf muscle pump. Eur J Appl Physiol Occup Physiol 1989; 59:39-47

17 Bjornberg J, Maspers M, Mellander S. Metabolic control of large-bore arterial resistance vessels, arterioles, and veins in cat skeletal muscle during exercise. Acta Physiol Scand 1989; 135:83-94

18 Schwartz LM, McKenzie JE. Adenosine and active hyperemia in soleus and gracilis muscle of cats. Am J Physiol 1990; 259:H1295-H1304

19 Corrigan TP, Kakkar VV. Early changes in the post-phlebitic limb: their clinical significance. Br J Surg 1973; 60:808-813

20 Qvarfordt P, Eklof B, Ohlin P, et al. Intramuscular pressure, blood flow, and skeletal muscle metabolism in patients with venous claudication. Surgery 1984; 95:191-195

21 Szmolenszky T, Mark B, Kiss T, et al. Ultrastructural damage of crural muscle in post-thrombotic syndrome. Acta Chir Hung 1984; 25:107-118

22 Makitie J. Muscle changes in patients with varicose veins. Acta Pathol Microbiol Scand [A] 1977; 85:864-868

23 McNair PJ, Stanley SN. Effect of passive stretching and jogging on the series elastic muscle stiffness and range of motion of the ankle joint. Br J Sports Med 1996; 30:313-317

24 Leng GC, Fowler B, Ernst E. Exercise for intermittent claudication. Cochrane Database Syst Rev 2000; (2): CD000990

25 Tan KH, De Cossart L, Edwards PR. Exercise training and peripheral vascular disease. Br J Surg 2000; 87:553-562

26 Remijnse-Tamerius HC, Duprez D, De Buyzere M, et al. Why is training effective in the treatment of patients with intermittent claudication? Int Angiol 1999; 18:103-112 


\section{Acute Effects of Exercise in Patients With Previous Deep Venous Thrombosis: Impact of the Postthrombotic Syndrome}

Susan R. Kahn, Laurent Azoulay, Andrew Hirsch, Michael Haber, Carla Strulovitch and Ian Shrier

Chest 2003;123;399-405

DOI: $10.1378 /$ chest.123.2.399

This information is current as of May 16, 2006

\begin{tabular}{|c|c|}
\hline $\begin{array}{l}\text { Updated Information } \\
\text { \& Services }\end{array}$ & $\begin{array}{l}\text { Updated information and services, including high-resolution } \\
\text { figures, can be found at: } \\
\text { http://www.chestjournal.org/cgi/content/full/123/2/399 }\end{array}$ \\
\hline References & $\begin{array}{l}\text { This article cites } 24 \text { articles, } 4 \text { of which you can access for free } \\
\text { at: } \\
\text { http://www.chestjournal.org/cgi/content/full/123/2/399\#BIBL }\end{array}$ \\
\hline Citations & $\begin{array}{l}\text { This article has been cited by } 1 \text { HighWire-hosted articles: } \\
\text { http://www.chestjournal.org/cgi/content/full/123/2/399\#othera } \\
\text { rticles }\end{array}$ \\
\hline Permissions \& Licensing & $\begin{array}{l}\text { Information about reproducing this article in parts (figures, } \\
\text { tables) or in its entirety can be found online at: } \\
\text { http://www.chestjournal.org/misc/reprints.shtml }\end{array}$ \\
\hline Reprints & $\begin{array}{l}\text { Information about ordering reprints can be found online: } \\
\text { http://www.chestjournal.org/misc/reprints.shtml }\end{array}$ \\
\hline Email alerting service & $\begin{array}{l}\text { Receive free email alerts when new articles cite this article } \\
\text { sign up in the box at the top right corner of the online article. }\end{array}$ \\
\hline Images in PowerPoint format & $\begin{array}{l}\text { Figures that appear in CHEST articles can be downloaded for } \\
\text { teaching purposes in PowerPoint slide format. See any online } \\
\text { article figure for directions. }\end{array}$ \\
\hline
\end{tabular}

A M E R I C A N C O L L E G E O F

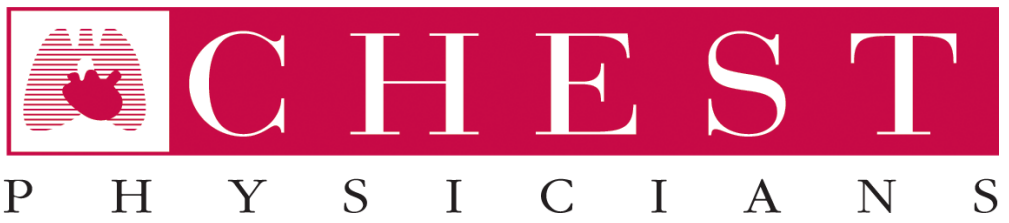

